\title{
Invariant Submanifolds in a Indefinite Trans-Sasakian Manifold
}

\author{
Nanditha S Matad \\ New Horizon College, Marathalli, Bangalore-560103, Karnataka, INDIA.
}

\begin{abstract}
In this paper, invariant submanifolds in a indefinite trans-Sasakian manifold are studied. Necessary and sufficient condition are given on submanifold of a indefinite trans-Sasakian manifold to be invariant submanifold.Here we shown that an invariant submanifold of a indefinite trans-Sasakian manifold is
\end{abstract} totally geodesic.

AMS Subject Classification: 53C15,53C17, 53C20, 53C25.

Keywords: Indefinite trans-Sasakian manifold, Invariant submanifold, Covariant differentiation, Bianchi identity, totally geodesic,

\section{Introduction}

In 1973 and 1974 B.Y.Chen and K. Ogive introduced geometry of submanifolds and totally real submanifolds in [1] and [2]. In [3] D.E Blair discussed contact manifold in Riemannian geometry in 1976. Light like submanifolds and hypersurfaces of indefinite sasakian manifolds introduced in 2007 and 2003 [4] and [5]. In 2010, F. Massamba introduced light like hypersurfaces in indefinite trans sasakian manifolds in [6]. In recent works many authors for example [7] C.S. Bagewadi and P.Venkatesha study trans sasakian manifolds, [8] Aysel Turgut Vanli and Ramazan sari study invariant submanifolds of trans sasakian manifolds. [9] Arindam Bhattacharya and Bandana Das study some properties of Contact CR-Submanifolds of an indefinite trans sasakian manifold. [10] B.Ravi and C.S. Bagewadi study invariant sub manifolds in a conformal K- Contact Riemannian manifold.

\section{Preliminaries}

Let $\bar{M}$ be an $(2 n+1)$-dimensional indefinite almost contact metric manifold with indefinite almost contact metric structure $(\phi, \xi, \eta, g)$ then they satisfies

$$
\begin{aligned}
\phi^{2} & =-I+\eta \otimes \xi, \\
\eta(\xi) & =1, \quad \phi \xi=0, \\
g(\phi X, \phi Y) & =g(X, Y)-\epsilon \eta(X) \eta(Y), \\
g(X, \xi) & =\epsilon \eta(X)
\end{aligned}
$$

where $\mathrm{X}, \mathrm{Y}$ are vector fields on $\overline{\mathrm{M}}$ and where $\epsilon=\mathrm{g}(\xi, \xi)= \pm 1$

An indefinite almost contact metric structure $(\phi, \xi, \eta, g)$ on $\mathrm{M}$ is called indefinite trans-Sasakian if

$$
\left(\bar{\nabla}_{X} \phi\right)(Y)=\alpha\{g(X, Y) \xi-\epsilon \eta(Y) X\}+\beta\{g(\phi X, Y) \xi-\epsilon \eta(Y) \phi X\}
$$

where $\alpha$ and $\beta$ are non zero scalar funtions on $\bar{M}$ of type $(\alpha, \beta) . \bar{\nabla}$ is a Riemannian connection on $\bar{M}$. In particular, an indefinite trans-Sasakian manifold is normal.

From above formula, one easily obtains

$$
\bar{\nabla}_{X} \xi=-\alpha \epsilon \phi X+\beta\{\epsilon X-\epsilon \eta(X) \xi\}
$$

Let $\mathrm{M}$ be an $(2 \mathrm{~m}+1)$ dimensional $(\mathrm{n}>\mathrm{m})$ manifold imbedded in $\overline{\mathrm{M}}$. The induced metric $\mathrm{g}$ of $\mathrm{M}$ is given by $\mathrm{g}(\mathrm{X}, \mathrm{Y})=\overline{\mathrm{g}}(\mathrm{X}, \mathrm{Y})$ for any vector fields $\mathrm{X}, \mathrm{Y}$ on $\mathrm{M}$.

Let $\mathrm{T}_{\mathrm{x}}(\mathrm{M})$ and $\mathrm{T}_{\mathrm{x}}(\mathrm{M})^{\perp}$ denote that tangent and normal bundles of $\mathrm{M}$ and $\mathrm{x} \varepsilon \mathrm{M}$. Let $\nabla \cdot \mathrm{x}$ denote the Riemannian connection on $\mathrm{M}$ determined by the induced metric $\mathrm{g}$ and $\mathrm{R}$ denote the Riemannian curvature tensor of $\mathrm{M}$. Then Gauss-Weingarten formula is given by 


$$
\begin{aligned}
& \bar{\nabla}_{X} Y=\nabla_{X} Y+B(X, Y), \\
& \bar{\nabla}_{X} N=-A_{N}(X)+D_{X} N
\end{aligned}
$$

for any vector fields $\mathrm{X}, \mathrm{Y}$ tangent to $\mathrm{M}$ and any vector field $N$ normal to $\mathrm{M}$, where $\mathrm{D}$ is the operator of covariant differentiation with respect to the linear connection induced in the normal bundle $\mathrm{T}_{\mathrm{x}}\left(\mathrm{M}^{\perp}\right)$. Both $\mathrm{A}$ and $\mathrm{B}$ are called the second fundamental forms of they satisfy

$$
\mathrm{g}(\mathrm{B}(\mathrm{X}, \mathrm{Y}), \mathrm{N})=\mathrm{g}\left(\mathrm{A}_{N}(\mathrm{X}, \mathrm{Y})\right) .
$$

A submanifold $M$ of $\bar{M}$ is said to be invariant if $\xi$ tangent to $M$ everywhere on $M$ and $\bar{\phi} X$ is tangent to $M$ for any tangent vector $\mathrm{X}$ to $\mathrm{M}$. An invariant submanifold $\mathrm{M}$ has the induced structure tensor $(\phi, \xi, \eta, \mathrm{g})$.

\section{Invariant Submanifolds in Indefinite Trans-Sasakian Manifold}

Let $\bar{M}$ be a $(2 n+1)$ dimensional indefinite trans-Sasakian manifold and $M$ a $(2 m+1)$ dimensional $(n>$ $\mathrm{m})$ manifold imbedded in $\overline{\mathrm{M}}$. For the second fundamental form $\mathrm{B}$ of an invariant submanifold $\mathrm{M}$ of a indefinite trans-Sasakian manifold. We define its covariant derivative $\left(\widetilde{\nabla_{X}} B\right)$ by

$$
\left(\bar{\nabla}_{X} B\right)(Y, Z)=D_{X}(B(Y, Z))-B\left(\nabla_{X} Y, Z\right)-B\left(Y, \nabla_{X} Z\right),
$$

where $\mathrm{X}, \mathrm{Y}, \mathrm{Z} \varepsilon \chi(\mathrm{M})$ - the set of all differential vector field on $\mathrm{M}$.

Then by $(2.7)$.We obtain

(3.2) $\bar{R}(X, Y) Z=R(X, Y) Z-A_{B(Y, Z)}(X)+A_{B(X, Z)}(Y)+\left(\bar{\nabla}_{X} B\right)(Y, Z)-\left(\bar{\nabla}_{Y} B\right)(X, Z)$

Lemma 3.1. If $M$ is an invariant submanifold of a indefinite trans-Sasakian manifold $\bar{M}$, then its second fundamental form $B$ satisfies $B(X, \xi)=0$, for any $X \varepsilon \chi(M)$.

Proof: Since $\bar{\xi}$ is tangent to $\mathrm{M}$ everywhere on $\mathrm{M}$, we have

$$
\bar{\nabla}_{X} \bar{\xi}=\bar{\nabla}_{X} \xi=\nabla_{X} \xi+B(X, \xi) .
$$

Since by equation,

$$
\bar{\nabla}_{X} \bar{\xi}=\bar{\nabla}_{X} \xi=-\alpha \epsilon \phi X+\beta\{\epsilon X-\epsilon \eta(X) \xi\}
$$

$\bar{\nabla}_{\mathrm{X}} \xi$ is tangent to $\mathrm{M}$ for any $\mathrm{X} \varepsilon \chi(\mathrm{M})$.

$$
\begin{aligned}
\bar{\nabla}_{X} \xi & =\nabla_{X} \xi+B(X, \xi) . \\
-\alpha \epsilon \phi X+\beta\{\epsilon X-\epsilon \eta(X) \xi\} & =\nabla_{X} \xi+B(X, \xi) .
\end{aligned}
$$

then by taking the normal parts of $(3.3)$ we get $\mathrm{B}(\mathrm{X}, \xi)=0$.

Lemma 3.2. Any invariant submanifolds $M$ with induced structure tensors of a indefinite trans-Sasakian manifold $\bar{M}$ is also indefinite trans-Sasakian manifold.

Proof: From (3.2) and lemma (3.1), we have

$$
\bar{R}(X, \bar{\xi}) \bar{\xi}=R(X, \xi) \xi+\left(\bar{\nabla}_{X} B\right)(\xi, \xi)-\left(\bar{\nabla}_{\xi} B\right)(X, \xi) .
$$

Again from equation From (3.1) and lemma (3.1), we get

$$
\left(\bar{\nabla}_{X} B\right)(\xi, \xi)=0,\left(\bar{\nabla}_{X} B\right)(X, \xi)=0 .
$$

Finally using From (3.5) in (3.4), we obtain

$$
\begin{aligned}
\bar{R}(X, \bar{\xi}) \bar{\xi} & =R(X, \xi) \xi+0+0, \\
R(X, \xi, \xi) & =-\alpha(\epsilon \eta(X) \xi-X)+\beta(\phi X) .
\end{aligned}
$$

Hence the lemma. 
Lemma 3.3. Let $M$ be an invariant submanifold of a indefinite trans-Sasakian manifold $\bar{M}$, then $\bar{R}(X, \xi) Y$ is tangent to $\mathrm{M}$ iff $\phi \mathrm{B}(\mathrm{X}, \phi \mathrm{Y})=\mathrm{B}(\mathrm{X}, \phi \mathrm{Y})$ for any $\mathrm{X}, \mathrm{Y} \varepsilon \chi(\mathrm{M})$.

Proof:

Then we have

$$
\begin{aligned}
\left(\bar{\nabla}_{X} \phi\right) Y & =\bar{\nabla}_{X} \phi Y-\phi\left(\bar{\nabla}_{X} Y\right) \\
& =\nabla_{X} \phi Y+B(X, \phi Y)-\phi\left(\nabla_{X}, Y\right)-\phi(B(X, Y)) \\
& =\left(\nabla_{X} \phi\right) Y+B(X, \phi Y)-\phi(B(X, Y))
\end{aligned}
$$

thus we get

$$
\alpha(g(X, Y) \xi-\epsilon \eta(Y) X)+\beta(g(\phi X, Y) \xi-\epsilon \eta(Y) \phi X)
$$$$
=\alpha(g(X, Y) \xi-\epsilon \eta(Y) X)+\beta(g(\phi X, Y) \xi-\epsilon \eta(Y) \phi X)+B(X, \phi Y)-\phi(B(X, Y))
$$

$$
B(X, \phi Y)=\phi(B(X, Y))
$$

Lemma 3.4. Let $M$ be invariant submanifold of the indefinite trans saskian manifold $M$ then,

$$
\bar{\nabla}_{X} B(Y, \xi)=-B\left(Y, \bar{\nabla}_{X} \xi\right)
$$

for any $\mathrm{X}, \mathrm{Y} \varepsilon \chi(\mathrm{M})$

Proof: By using Lemma 3.1 we get

Then, we have

$$
\bar{\nabla}_{X} B(Y, \xi)=\nabla_{X} B(Y, \xi)-B\left(\nabla_{X} Y, \xi\right)-B\left(Y, \bar{\nabla}_{X} \xi\right)
$$

$$
\bar{\nabla}_{X} B(Y, \xi)=-B\left(Y, \bar{\nabla}_{X} \xi\right)
$$

Theorem 3.1. Let $M$ be an invariant submanifold of an indefinite trans sasakian manifold $\bar{M}$. Then $B$ is parallel if and only if $\mathrm{M}$ is totally geodesic.

Proof: Suppose that B is parallel. For each $\mathrm{X}, \mathrm{Y} \varepsilon \chi(\mathrm{M})$ and using lemma 3.4 we get,

$$
\nabla_{X} B(Y, \xi)=0
$$

$$
B\left(Y, \nabla_{X} \xi\right)=0
$$

BY equation $(2.1)$, we have

$$
\bar{\nabla}_{X} \xi=-\alpha \epsilon \phi X+\beta\{\epsilon X-\epsilon \eta(X) \xi\}
$$

$$
\bar{\nabla}_{X} \xi=-\alpha \epsilon \phi X-\beta \epsilon \phi^{2}
$$

Hence

$$
\begin{array}{r}
B\left(Y,-\alpha \epsilon \phi X-\beta \epsilon \phi^{2} X\right)=0 \\
-\alpha B(Y, \epsilon \phi X)-\beta B\left(Y, \epsilon \phi^{2} X\right)=0
\end{array}
$$

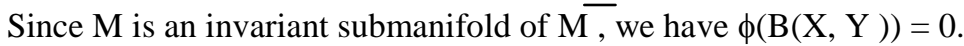

From Lemma 3.3 it follows that

$$
\phi(B(X, Y))=B(X, \phi Y)=0
$$

Then we get

hence it follows that

$$
\beta B\left(Y, \epsilon \phi^{2} X\right)=0
$$

$$
B(Y,-X+\epsilon \eta(X) \xi)=0
$$

so

$$
\mathrm{B}(\mathrm{Y}, \mathrm{X})=0
$$

viceversa let $\mathrm{M}$ is totally geodesic, Then $\mathrm{B}=0$, for all $\mathrm{X}, \mathrm{Y}, \mathrm{Z} \varepsilon \mathrm{TM}$.

thus we have $\nabla \cdot \mathrm{B}=0$

$$
\left(\bar{\nabla}_{X} B\right)(Y, Z)=D_{X}(B(Y, Z))-B\left(\nabla_{X} Y, Z\right)-B\left(Y, \nabla_{X} Z\right)=0
$$




\section{References}

[1]. B.Y Chen, Geometry of submanifolds, M.Dekker,Newyork, 1973

[2]. B.Y.Chen and K.Ogive , on totally real submanifolds, Transaction of the Amer.Math.Society, 193(1974), 257-266

[3]. D.E. Blair, Contact manifold in Riemannian Geometry, Lecture Notes in Mathematics, 509, Springer-Verlag, Berlin, 1976

[4]. K.L.Duggal and B.Sahin, Lightlike submanifolds of Indefinite sasakian manifolds, International Journal of mathematics and Matematical Sciences, Hindawi Publishing Corporation (2007),1-22

[5]. T.H.Kang,S.D.Jung,B.H.Kim,H.K.pak and J.S.Pak, Light like hypersurfaces of indefinite sasakian manifolds, Indian Journal of Pure and Applied mathematics, Vol.34,no.9(2003),1369-1380.

[6]. F. Massamba, light like hypersurfaces in indefinite trans sasakian manifolds , IC,044(2010),1-29.

[7]. C.S. Bagewadi and P.Venkatesha, Torseforming vector field in a 3-dimentional Trans sasakian manifold , Differ.Geom.Dyn.Syst.,8(2006),23-28.

[8]. Aysel Turgut Vanli and Ramazan sari, Contact CR-Submanifolds of an indefinite trans sasakian manifold., Differ.Geom.Dyn.Syst.,12(2010),277-288

[9]. Arindam Bhattacharya and Bandana Das, invariant submanifolds of trans sasakian manifolds, Int.J.Contemp.Math.Science,Vol 6,2011, no. $26,1271-1282$

[10]. C.S. Bagewadi and B. Ravi, Invariant submanifolds of a conformal k-contact manifold, Indian J. Pure and Appl. Math., 20 (1989), 1119-1125. 